International Journal of Soft Computing 5 (2): 62-66, 2010

ISSN: $1816-9503$

(C) Medwell Journals, 2010

\title{
Neural Network Prediction of Electromagnetic Field Strength in Hybrid Micro-Grid System
}

\author{
${ }^{1}$ Ahmed M.A. Haidar, ${ }^{2}$ Tbrahim A. Ahmed and ${ }^{1}$ Ahmed N. Abdalla \\ ${ }^{1}$ Faculty of Electrical and Electronics Engineering, University Malaysia Pahang, \\ Kuantan, Pahang, Malaysia \\ ${ }^{2}$ Hodeidah University, Yemen, Saudi Arabia
}

\begin{abstract}
Location of any Hybrid Micro-Grid System requires efficiently prediction of the electromagnetic field strength. This study proposes a novel Electromagnetic Field Strength (EFS) predication based on Probabilistic Neural Network (PNN). Learning data sets have been generated using Electromagnetic Transients Program EMTP. The PNN model has three input nodes representing the Switching Distance, Busbar Interference Voltage and Current waveforms, the output node representing the EFS. Testing datasets have deliberately been chosen outside the region of the learning datasets so as to check the performance of the neural network. The results indicate that the proposed technique can be used successfully to detect the maximum Electromagnetic Field Strength Level at any Location.
\end{abstract}

Key words: Probabilistic neural network, electromagnetic interference, hybrid micro-grid system, electromegnatic field, dalaset, Malaysia

\section{INTRODUCTION}

Micro-Grid is one of the expected local power supply system that consists of distributed generators, loads, power storage devices, heat recovery equipments and power electronics equipments. Recently, distributed generation units are increasingly being used because of their economical and environmental benefits compared to the use of large power plants. Many distributed power sources such as wind turbines, photovoltics and fuel cells do not generate a $50 \mathrm{~Hz}$ voltage so they require a voltage-source inverter as an interface to the grid. All power electronics equipments generate and emit unwanted electrical signals (Electromagnetic interference noise) that can lead to performance degradation of other electrical/electronic equipments (Liang and Dou, 2009).

The undesirable Electromagnetic Interference (EMI) effects are generated high frequency conducted and radiated EMI noise and draw distorted line currents due to the sharp edges of the switching waveforms with high $\mathrm{dv} / \mathrm{dt}$. The other effects of EMI come from following aspects such as routine connect or disconnect switch and breaker operations, lightning and short-circuiting in power system, partial discharge, electric field or magnetic field created by high voltage busbar, radiation created by automatic devices and radio devices. No utility scale renewable energy system shall be installed in any location within the line of sight of an existing microwave communications link where operation of the energy system is likely to produce EMI in the link's operation unless the interference is insignificant (Vandoorn et al., 2009).

Due to the rapid development of power electronics connected to the renewable energy source in many countries, the PV station is built on many places of remote area residential, communal building. With the increased utilization of distributed power, the power quality problems are expected to increase such as distortion of sinusoidal voltage and current waveform caused by harmonics from grid connected inverter which is considered as one of the major problems in electrical power system (Khamphakdi et al., 2006; Wang et al., 2009). Accordingly, an accurate and fast technique is needed to predict EFS level for a given system can be a very useful solution.

Few researches focused on the topic of effect of electromagnetic interference in term of neural networks application for fast and accurate detection. Presently, artificial neural networks have shown great promise as new techniques for solving critical problems in deferent engineering fields due to its ability to do parallel data processing, high accuracy and fast response (Wahab et al., 2007; Jadid and Jalilzadeh, 2005; Song et al., 2005; Hong and Chen, 2007; Doorman et al., 2006). In this research, an intelligent method based on

Corresponding Author: Ahmed M.A. Haidar, Faculty of Electrical and Electronics Engineering, University Malaysia Pahang, Kuatan, Pahang, Malaysia 
PNN is presented to predict the EFS in the Micro grid system. The input data used to identify the level of EFS are Switching Distance, Busbar Interference Voltage and Current waveforms. The output of the PNN is the prediction of the EFS level whether the level is low or medium or high.

\section{MATERIALS AND METHODS}

System description: The system architecture employed in the hybrid micro-grid system is AC Coupled where the renewable energy sources and the conventional diesel generators all feed into the $\mathrm{AC}$ side of the network as shown in Fig. 1. All power generators feed DC power into a battery.

Each component has therefore to be equipped with an individual charge controller and in the case of a diesel generator with a rectifier. During periods of low electricity demand the diesel generator is switched off and the load can be supplied from renewable sources together with stored energy. AC power delivered to the load is converted from $\mathrm{DC}$ to regulated $\mathrm{AC}$ by an inverter (Vandoorn et al., 2009; Dehbonei, 2003).

Distributed power and in particular PV inverters are currently based on single-phase self commutated voltage-source inverters for individual households. This inverter consists of different power stages with highfrequency and line-frequency transformers or even transformer less design. For increasing efficiency reasons, transformers less topologies have been used in (Khamphakdi et al., 2006), isolation transformer is required for safety requirement.

In practice, switching frequency $20-500 \mathrm{kHz}$ is used in different power stages with mainly Metal Oxide Semiconductor Field Effect Transistors (MOSFETs) and Insulated Gat Bipolar Transistors (IGBTs) as switching elements for this PV inverter (Khamphakdi et al., 2006). Usually, in some cases is used single-stage Pulse

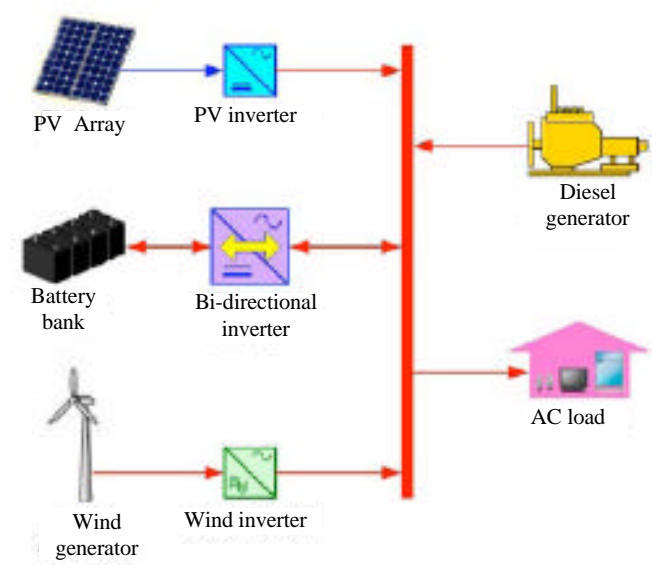

Fig. 1: Schematic diagram of Hybrid system
Width Modulated (PWM) DC-AC converter topology (H-Bridge), directly coupled to the grid via a lowfrequency isolation transformer and filters as shown in Fig. 2.

Problem formulation: Electromagnetic Interference, normally divided into two kinds of interference, conducted interference and radiated interference. Mainly conducted interference generated by electronic devices interfere with other devices through the conductive medium or through the public power line, radiated interference is the interference generated by electronic devices coupled to each other through space.

An example of switch operation can be simplified using the equivalent circuit as shown in Fig. 3. In this circuit, the main components are capacitor $\mathrm{C}$ which is located for the no load busbar, Resistance (R), inductance (L) and the isolated switch $(\mathrm{K})$. Power system is a power sine wave, however each time the electric arc restrik takes only a very short time in the insulating layer when it moves from on to off position. In this period of time, the power supply voltage change is very little. Therefore, the power supply can be considered as a DC power supply for each time when the arc restriking (Brdys et al., 1999).

When switch is turned on, the moving contact moves to the static contact at a certain speed and the distance between this two parts gradually shrinking. While the potential difference between the air insulation is sufficient enough, the first arc is produced. Once the flashover begins, the capacitor will be rapidly charged by the current through the switch contact. After a brief time of oscillation process, potential of the busbar change from initial state zero to instantaneous value of the power supply voltage.

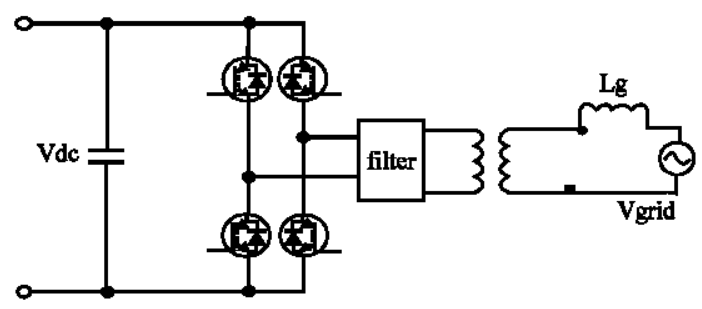

Fig. 2: Grid connected inverter topology

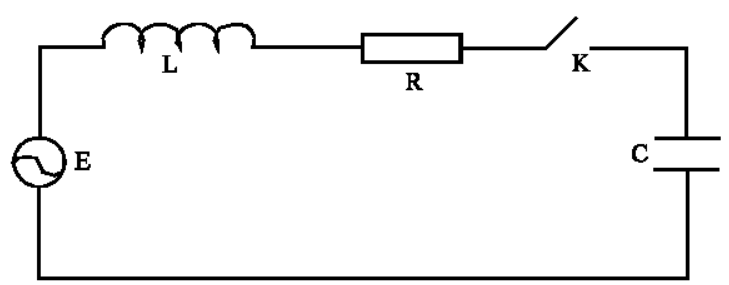

Fig. 3: Simplified equivalent circuit of switch operation 
The capacitor is charged and the voltage between the switch contacts becomes zero, thus the arc between the switch contacts will be broken off at this time, if the charge leakage is neglected, the potential of the busbar will maintain the value as the arc was blew out. Consequently, the potential between the switch contacts frequently will be changed as the power supply voltage become change. The voltage across the switch contacts increase again until the insulating layer is breakdown again. Bus potential reoscillating and the instantaneous value of the power supply voltage and arc is then blown out. Thus, the arc restrik blows out again and again, until the moving contact and static contact touch each other.

The oscillation frequency $f_{\mathrm{cm}}$ of the equivalent can be deriving from the total series inductance $\mathrm{L}$ and capacitance $\mathrm{c}$ by:

$$
\mathrm{f}_{\mathrm{cm}}=\frac{1}{2 \pi \sqrt{\mathrm{LC}}}
$$

The power supply and switch may be described as an equivalent voltage source $\mathrm{v}(\mathrm{t})$ with switching time, $\Delta \mathrm{t}=\mathrm{t}_{\mathrm{i}}-0$, thus the $\mathrm{KVL}$ equation around the loop by (Zhong et al., 1996):

$$
\mathrm{Ri}+\mathrm{L} \frac{\mathrm{di}}{\mathrm{dt}}+\frac{1}{\mathrm{C}} \int_{0}^{\mathrm{t}} \mathrm{idt}=\mathrm{V}(\mathrm{t})=\mathrm{V}^{\prime} \mathrm{t}
$$

Solving for $i(t)$ with initial condition $i(0)=0$ and $\mathrm{V}_{0}(0)=0$, the initial current is:

Where:

$$
i=C V\left[1-\sqrt{1+\left(\frac{\beta}{\omega_{1}}\right)^{2}} e^{-\beta t} \cos \left(\omega_{1} t-\theta\right)\right.
$$

$2 \beta=\mathrm{R} / \mathrm{L}$

$\omega_{0}^{2}=1 / \mathrm{LC}$

$\omega^{2}=\omega_{0}^{2}-\beta^{2}$

$\tan \theta=\beta / \omega_{1}$

The oscillatory current with an amplitude depending on both the initial condition and the supply voltage $V_{0}$ and reactance $\omega \mathrm{L}$, lasting a period of about 3-4 times of $\beta / \omega_{1}$ therefore, oscillatory current is obtained as follows:

$$
\left.i=\sqrt{\left(\frac{V_{0}-V_{0}\left(t_{1}\right)}{\omega_{1} L}\right)^{2}+i\left(t_{1}\right)^{2}\left[1+\left(\frac{\beta}{\omega_{1}}\right)^{2}\right.}\right] e^{-\beta t} \sin \left(\omega_{1} t+\phi\right)^{(4)}
$$

PNN implementation for EFS predication: PNN which is a class of Radial Basis Function (RBF) network is useful for automatic pattern recognition, nonlinear mapping and estimation of probabilities of class membership and likelihood ratios. It is a direct continuation of the work on

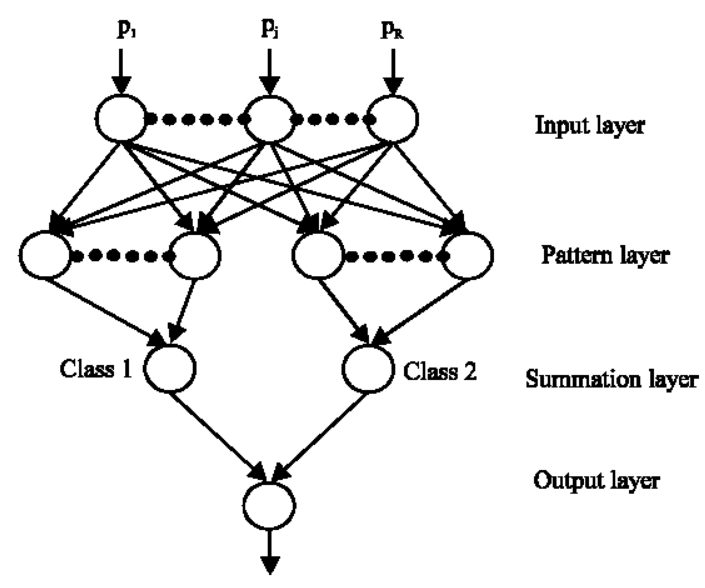

Fig. 4: PNN architecture

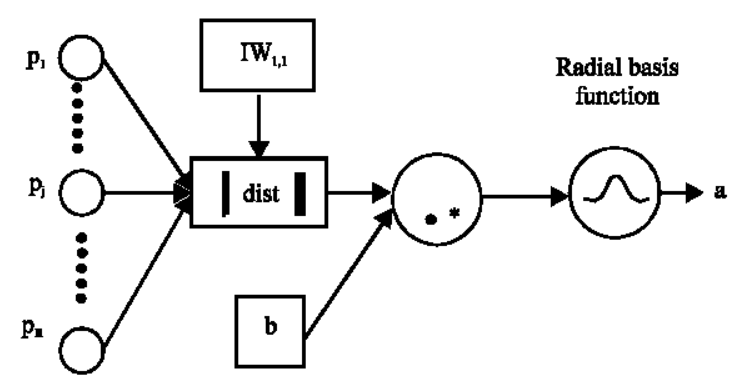

Fig. 5: PNN pattern layer

Bayes classifiers in which it is interpreted as a function that approximates the probability density of the underlying example distribution. The PNN consists of nodes with four layers namely input, pattern, summation and output layers as shown in Fig. 4.

The input layer consists of merely distribution units that give similar values to the entire pattern layer. For this research, $\mathrm{RBF}$ is used as the activation function in the pattern layer and the inputs are Switching Distance, Busbar Interference Voltage and Current waveforms.

Figure 5 shows the pattern layer of the PNN (Song et al., 2005). The $\|$ dist $\|$ box shown in Fig. 5 subtracts the input weights $\mathrm{IW}_{1.1}$, from the input vector, $\mathrm{p}$ and sums the squares of the differences to find the Euclidean distance. The differences indicate how close the input is to the vectors of the training set. These elements are multiplied element by element with the bias, b using the dot product $\left({ }^{*}\right)$ function and sent to the radial basis transfer function (Specht, 1992). The output a is given as:

$$
\mathrm{a}=\operatorname{radbas}\left(\left\|\mathrm{IW}_{1.1}-\mathrm{P}\right\| \mathrm{b}\right)
$$


where, radbas is the radial basis activation function which can be written in general form as:

$$
\operatorname{radbas}(n)=\mathrm{e}^{\mathrm{n}^{2}}
$$

The training algorithm used to train the RBF is the orthogonal least squares method which provides a systematic approach to the selection of RBF centers. The summation layer shown in Fig. 4 simply sums the inputs from the pattern layer which correspond to the category from which the training patterns are selected as either class 1 or class 2. Finally, the output layer of the PNN is a binary neuron that produces the classification decision. As for this research, the classification is either class 1 for low EFS level or class 2 for high EFS level.

\section{RESULTS AND DISCUSSION}

The results obtained from the PNN for Electromagnetic Field Strength predication are presented in this section. Initially, the EMTP has been used to perform a transient analysis to get voltage and current profiles and for the determinations of EMI. From Figure 6 and 7 , it is shown that simple simulation methods can give a useful insight of the EMI during the switching process on the electrical system connected to Hybrid

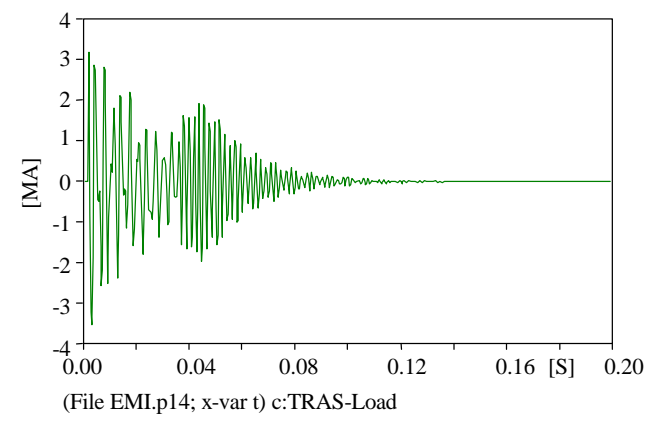

Fig. 6: The effect of switching on current profile due to the EMI

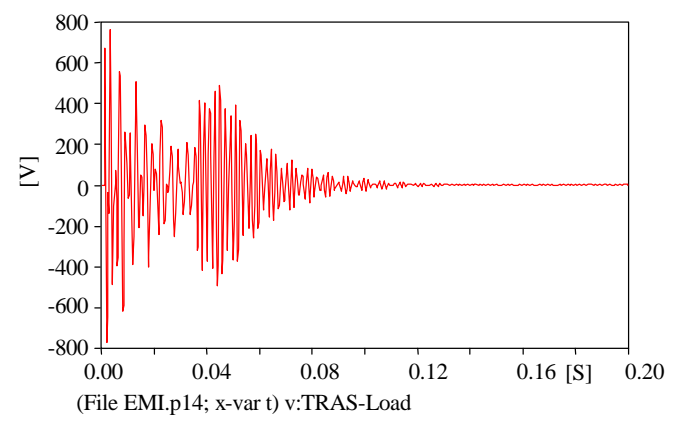

Fig. 7: The effect of switching on voltage profile due to the EMI
Micro-Grid. In addition, the waveforms attenuation of the voltage and current can be noted as shown in the Fig. 6 and 7. Here, the voltage and current waveforms are increased and then these values after a period of time are decreased. The EMI effects generate a high frequency conducted and radiated EMI noise at the busbar due to the sharp edges switching waveforms with a high $\mathrm{dv} / \mathrm{dt}$ and $\mathrm{di} / \mathrm{dt}$, this frequency causes waveform distortion of voltage and current.

In the switching process, the distance between the contacts narrowing, the potential difference between two contacts gradually decreased. So in one cycle of the power frequency, the time of arc restrike gradually increased. The busbar voltage becomes more and more dense ladder like waveforms. Similarly, when the switch is turned off it just inverts entry almost all of the above-mentioned process, the difference is that when the last time flashover disconnected, it will leave a residual charge in the busbar.

A summary of obtained results for Electromagnetic Field Strength predication using PNN are shown in Table 1 , the results are evaluated in terms of absolute error which is the difference between the desired output determined by EMTP analysis and the PNN output.

\begin{tabular}{|c|c|c|c|c|}
\hline Test case & PNN EFS & Desired EFS & Absolute error & EFS level \\
\hline 1 & 1 & 1 & 0 & Low strength \\
\hline 2 & 2 & 2 & 0 & Mediumstrength \\
\hline 3 & 3 & 3 & 0 & High strength \\
\hline 4 & 1 & 1 & 0 & Low strength \\
\hline 5 & 2 & 2 & 0 & Mediumstrength \\
\hline 6 & 3 & 3 & 0 & High strength \\
\hline 7 & 1 & 1 & 0 & Low strength \\
\hline 8 & 2 & 2 & 0 & Mediumstrength \\
\hline 9 & 3 & 3 & 0 & High strength \\
\hline 10 & 1 & 1 & 0 & Low strength \\
\hline 11 & 1 & 1 & 0 & Low strength \\
\hline 21 & 3 & 3 & 0 & High strength \\
\hline
\end{tabular}

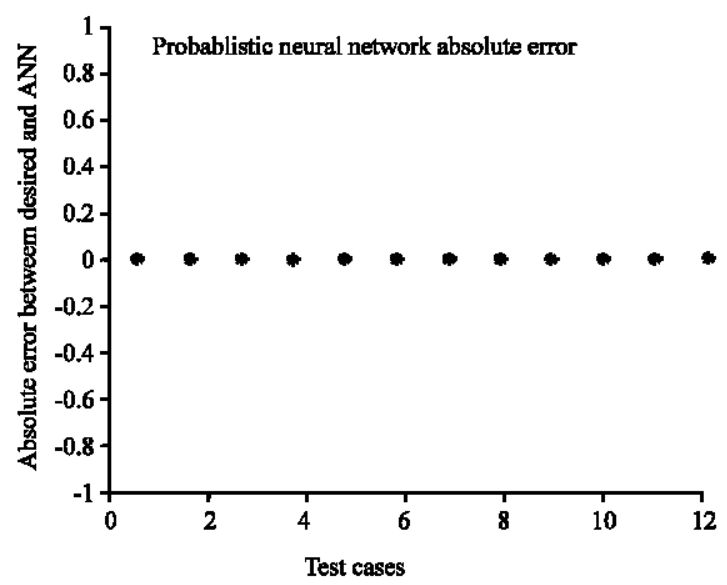

Fig. 8: The accuracy of the PNN for testing data 
Refereeing to Table 1, Column 4 shows the absolute error of the PNN for each test case. From the testing results, it is noted that in terms of absolute errors, the PNN is considered to predict the EFS of the Hybrid Micro-Grid System accurately as shown from Table 1 and shown graphically in Fig. 8. Based on these results, it can be concluded that the PNN is more fast and accurate in predicting the EFS level.

\section{CONCLUSION}

This study presents a fast prediction of Electromagnetic Field Strength on hybrid Micro-grid System using Probabilistic Neural Network. Constructed models of switching operations are presented and discussed for some key components of the input and output data. The performance of the PNN has been evaluated in term of absolute error and the results show that PNN can be used as a tool to provide fast information about the conditions of the hybrid system during EMI. Furthermore, the results obtained from PNN show the level of EFS on the electric and electronic components of hybrid system. The fast prediction of EFS using PNN provides guidance for power system designers to select the optimal location of the PV/Wind/Diesel Micro-grid System.

\section{REFERENCES}

Brdys, C., J.P. Toumazet, G. Velleaud and S. Servant, 1999. Study of the low-voltage electric breaking arc restrike by means of an inverse method. IEEE Trans. Plasma Sci., 27: 595-603.

Dehbonei, H., C.V. Nayar and L. Chang, 2003. A new modular hybrid power system. Proceedings of IEEE International Symposium on Industrial Electronics, (ISIE'03), Rio de Janeiro, Brazil, pp: 985-990.

Doorman, G., K. Uhlen, G. Kjolle and E. Huse, 2006. Vulnerability analysis of the Nordic power system. IEEE Trans. Power Syst., 21: 402-410.

Hong, Y.Y. and B.Y. Chen, 2007. Hong, Y.Y. and B.Y. Chen, 2007 Locating switched capacitor using wavelet transform and hybrid principal component analysis network. IEEE Trans. Power Delivery, 22: 1145-1152.
Jadid, S. and S. Jalilzadeh, 2005. Application of neural network for contingency ranking based on combination of severity indices. Proceedings of World Academy of Science, Engineering and Technology, Nov. 2005, Tokyo, Japan, pp: 173-176.

Khamphakdi, P., V. Tarateeraseth, K. Karanun and W. Khan-ngern, 2006. The conducted electromagnetic interference of small grid connected inverter to power system. Proceedings of IEEE Conference on Electromagnetic Compatibility, (CEC'06), Thailand, pp: 654-657.

Liang, Z. and H. Dou, 2009. Analysis model of electromagnetic interference flowing in a substation cable. Proceedings of IEEE Conference on Power and Energy Engineering, March 27-31, Wuhan, China, pp: 3-3.

Song, Y., G. Bu and R. Zhang, 2005. A fast method for probabilistic reliability assessment of bulk power system using fsom neural network as system states filters. Proceedings of IEEE/PES Transmission and Distribution Conference and Exhibition: Asia and Pacific, Dec. 5, Dalian, China, pp: 1-6.

Specht, D.F., 1992. Enhancements to probabilistic neural networks. Int. Joint Conf. Neural Networks, 1: 525-532.

Vandoorn, T., B. Renders, F. de Belie, B. Meersman and L. Vandevelde, 2009. A voltage-source inverter for microgrid applications with an inner current control loop and an outer voltage control loop. Proceedings of International Conference on Renewable Energies and Power Quality, (ICREPQ'09), Valencia, Spain, pp: 1-6.

Wahab, N.I.A., A. Mohamed and A. Hussain, 2007. Transient stability assessment of a power system using PNN and LS-SVM methods. J. Applied Sci., 7: $3208-3216$.

Wang, L., R. Liu and H. Huang, 2009. Electromagnetic interference modeling research on the electrical machine and converter systems. Proceedings of Electromagnetics Research Symposium, March 23-27 Beijing, China, pp: 235-238.

Zhong, E., T.A. Lipo, J.R. Jaeschke and D. Gritter, 1996. Analytical estimation and reduction of conducted EMI emissions in high power PWM inverter drives. Proceedings of IEEE Conference on Power Electronic, Jun. 23-27 Baveno, pp: 1169-1175. 